\title{
Erratum: Model for the metal-insulator transition in graphene superlattices and beyond [Phys. Rev. B 98, 045103 (2018)]
}

\author{
Noah F. Q. Yuan and Liang Fu
}

(Received 18 July 2018; published 2 August 2018)

DOI: 10.1103/PhysRevB.98.079901

In the caption of Fig. 5, parameters for (c) should be $t_{1}=2, t_{1}^{\prime}=0, t_{2}=0.05, t_{2}^{\prime}=0.2, \mu=0$, and parameters for (d) should be the same as (c) except $t_{1}^{\prime}=0.2$. This change is made only within numerical parameters and does not affect the rest of the original paper.

In Eq. (20), $t_{2}, t_{2}^{\prime}$ should be $2 t_{2}, 2 t_{2}^{\prime}$ and hence $H_{2}(\boldsymbol{k})$ will be modified as

$$
H_{2}(\boldsymbol{k})=t_{2}\left(6-\frac{9 A^{2}}{2}|\boldsymbol{k}|^{2}\right)+\lambda\left(k_{+}^{3}+k_{-}^{3}\right) \tau_{z},
$$

where $\lambda=-\frac{3 \sqrt{3}}{8} A^{3} t_{2}^{\prime}$. This change is made only for this equation and does not affect the rest of the original paper.

In the last sentence of of paragraph 1, right column of page 4, , “...on the A sublattice has its maxima at $\mathrm{AB}$ spots on layer 1 and at BA spots on layer 2, while the component on the B sublattice has its maxima on BA spots on layer 1 and AB spots on layer 2", interchange layer 1 and layer 2. Correspondingly in the sentences near the end of the following paragraph, "hence must be located at AB spots on layer 1 and BA spots on layer 2" and "peaked at BA spots on layer 1 and AB spots on layer 2", layer 1 and layer 2 also need to be interchanged. These changes are made so that the descriptions in those two paragraphs are consistent with Eq. (7) of the main text, and they will not affect the rest of the original paper.

We thank Guangkun Liu from Beijing computational science research center for pointing out the first problem. 